\title{
Utilidad del Doppler transcraneal en la resucitación de la parada cardíaca
}

\author{
J.A. Álvarez-Fernández ${ }^{\mathrm{a}, *}$, M.M. Martín-Velasco ${ }^{\mathrm{b}}$, J.C. Igeño-Cano $^{\mathrm{b}}$ y R. Pérez-Quintero ${ }^{\mathrm{a}}$ \\ aUnidad de Neurosonología y Hemodinámica Cerebral, Hospital Hospiten-Rambla, \\ Santa Cruz de Tenerife, Islas Canarias, España

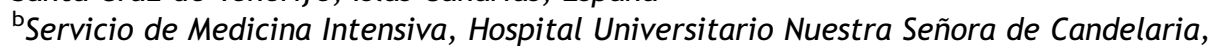 \\ Santa Cruz de Tenerife, Islas Canarias, España
}

Recibido el 30 de julio de 2009; aceptado el 17 de diciembre de 2009

Disponible en Internet el 7 de marzo de 2010

\section{PALABRAS CLAVE \\ Resucitación; \\ Síndrome posparada \\ cardíaca; \\ Ultrasonidos; \\ Doppler transcraneal}

\section{KEYWORDS}

Resuscitation;

Post cardiac arrest

syndrome;

Ultrasounds;

Transcranial Doppler

\begin{abstract}
Resumen
Durante la resucitación cardiopulmonar y tras la recuperación de la circulación espontánea, una perfusión tisular efectiva determina el pronóstico final. La ultranosografía Doppler transcraneal (DTC) registra la velocidad y la pulsatilidad del flujo sanguíneo cerebral y permite realizar análisis hemodinámicos «latido a latido». Durante la resucitación cardiopulmonar la velocidad sistólica máxima alcanzada refleja la perfusión cerebral en cada compresión torácica. Tras la recuperación de la circulación espontánea, la persistencia después de $2 \mathrm{~h}$ de un patrón arterial cerebral hipodinámico (baja velocidad media y alta pulsatilidad en la DTC) pronostica mala recuperación neurológica. La presencia, precoz o tardía, de un patrón de DTC hiperémico (alta velocidad media y baja pulsatilidad) se asocia a mal pronóstico por evolución a hipertensión intracraneal; su aparición durante la fase de recalentamiento debería llevar a reinstaurar la hipotermia terapéutica La coincidencia de arterias con patrones hipodinámicos con otras normales 0 hiperdinámicas indica focos de hipoperfusión que son predictores de ictus. (c) 2009 Elsevier España, S.L. y SEMICYUC. Todos los derechos reservados.
\end{abstract}

\section{Transcranial Doppler ultrasonography usefulness in cardiac arrest resuscitation}

\begin{abstract}
An effective tissue perfusion has decisive influence on the final prognosis both during cardiopulmonary resuscitation (CPR) and after recovery of spontaneous circulation (ROSC). The transcranial Doppler ultranosography (TCD) examines the velocity and pulsatility of cerebral blood flow, making it possible to perform "beat to beat" hemodynamic analysis. During CPR, TCD peak systolic velocity reflects cerebral perfusion of the chest compressions. Beyond 2 hours after ROSC, persistence in the cerebral arteries of a
\end{abstract}

\footnotetext{
*Autor para correspondencia.

Correo electrónico: UNSyHC@gmail.com (J.A. Álvarez-Fernández).
} 
hemodynamic TCD pattern (low velocities with high pulsatilities) predicts poor neurological prognosis. Early or delayed presence of a hyperemic TCD pattern (high velocities with low pulsatilities) is associated conclusively with evolution to intracranial hypertension and its appearance during the rewarming process should lead to immediate return to therapeutic hypothermia. The coincidence of hypodynamic cerebral arteries and others with normal or hyperemic TCD patterns may indicate the presence of focal hypoperfusion that could predict stroke after ROSC.

(c) 2009 Elsevier España, S.L. and SEMICYUC. All rights reserved.

\section{Introducción}

De cada 100 pacientes tratados en parada cardíaca por equipos de emergencias extrahospitalarios se logra resucitar sólo a la mitad inicialmente, 25 llegan a ingresarse en una unidad de cuidados intensivos o coronarios (UCI/UCC) y a 10 se les da de alta vivos del hospital ${ }^{1}$. Esta situación es ligeramente más favorable en el ambiente hospitalario, con un promedio del $37 \%$ de ingresos en la UCI/UCC y del $18 \%$ de altas hospitalarias ${ }^{2}$.

La realización ininterrumpida de una resucitación cardiopulmonar (RCP) de alta calidad aumenta la probabilidad de recuperación de la circulación espontánea (RCE) tras la parada cardíaca $^{3}$, y la hipotermia terapéutica es efectiva tras la RCE ${ }^{4}$. En ambos casos influirá en el pronóstico final el mantenimiento de una perfusión tisular efectiva, pero esta resucitación "orientada hacia el flujo sanguíneo» requiere sistemas sensibles y fiables de monitorización ${ }^{5}$. Optimizar la perfusión tisular sistémica es posible mediante la información obtenida con la oximetría del pulso, la esfigmomanometría, la capnografía u otras más complejas ${ }^{6,7}$. Sin embargo, el conocimiento real del flujo sanguíneo cerebral (FSC) ha tenido hasta fechas recientes una importante limitación por la complejidad de las técnicas empleadas para su medición (habitualmente tomografía de emisión de fotón único o tomografía de emisión de positrones) que tienen escasa aplicabilidad fuera del ambiente de los laboratorios de experimentación ${ }^{8}$.

La ultranosografía Doppler transcraneal (DTC) mide la velocidad del FSC «latido a latido» y puede analizar sus características en las diferentes fases de la resucitación de la parada cardíaca, con una sencillez y portabilidad que permiten su aplicación en la práctica clínica ${ }^{9,10}$. En este trabajo se aboga por el uso de la DTC en resucitación sobre la base de la evidencia científica disponible.

\section{Análisis ultrasonográfico de la hemodinámica cerebral}

Las técnicas directas de medición del FSC (gases inertes, microesferas o isótopos radiactivos, tomografía de emisión de positrones o tomografía de emisión de fotón único, etc.) tienen gran complejidad y escasa aplicabilidad asistencial en resucitación. Sin embargo, las características principales de la circulación sanguínea intracraneal pueden analizarse de forma no invasiva mediante ultrasonografía Doppler espectral de las arterias del polígono de Willis, obtenida mediante máquinas específicas («DTC») o mediante ecógrafos convencionales («ultrasonografía dúplex transcraneal») ${ }^{11,12}$.
La DTC mide, en cada latido de las arterias cerebrales, la velocidad del flujo sanguíneo sistólica (VS) y diastólica (VD), y calcula la velocidad media $(\mathrm{Vm})(\mathrm{Vm}=[\mathrm{VS}+2 \mathrm{VD}] / 3)$ y el índice de pulsatilidad ([VS-VD]/Vm). Mediante combinación de las Vm y los índices de pulsatilidad (IP) registrados pueden identificarse 5 patrones de velocidad de flujo que representan otras tantas posibilidades hemodinámicas (fig. 1) ${ }^{13}$. La DTC determina el FSC de forma indirecta, ya que la velocidad registrada es el cociente entre el FSC y el área del vaso que atraviesa; como el diámetro de los vasos del polígono de Willis se mantiene constante, incluso en condiciones extremas de hipoventilación/hiperventilación o hipotensión/hipertensión, los cambios en la velocidad del FSC reflejarán de forma fiel los producidos en el propio $\mathrm{FSC}^{14,15}$.

\section{Daño cerebral en la parada cardíaca}

El daño cerebral es causa común de morbimortalidad tras recuperar una parada cardíaca ${ }^{16}$. Durante la parada cardíaca el daño cerebral se produce por la ausencia de FSC y, por lo tanto, por isquemia-anoxia. Al inicio de la parada cardíaca con ritmos de taquicardia ventricular sin pulso o de fibrilación ventricular, se ha comprobado mediante DTC que se sigue conservando el FSC, probablemente por la vasodilatación inducida por anoxia con incremento en la presión crítica de cierre vascular, y no se produce consecuencia alguna si la situación es inmediatamente revertida (por ejemplo, con un desfibrilador implantado $)^{17}$.

Tras la RCE se identifican 5 intervalos determinantes del «síndrome posparada cardíaca»: inmediato, desde la RCE hasta transcurridos $20 \mathrm{~min}$; precoz, entre $20 \mathrm{~min}$ y $12 \mathrm{~h}$ tras la RCE; intermedio, entre $12-32 \mathrm{~h}$ tras la RCE; recuperación, transcurridas más de $72 \mathrm{~h}$ tras la RCE, y rehabilitación, desde el alta de la unidad de cuidados críticos ${ }^{18}$. Estos intervalos se superponen con las 4 fases fisiopatológicas descritas clásicamente como disfunción de todos los órganos y sistemas en las primeras $12 \mathrm{~h}$ tras la parada cardíaca (fase I); recuperación circulatoria con persistencia de la disfunción cerebral y metabólica entre las 12 - $24 \mathrm{~h}$ (fase II); normalización progresiva del funcionamiento de todos los órganos y sistemas transcurridas $72 \mathrm{~h}$ (fase III), y aparición de complicaciones diferidas superado este tiempo (fase IV) ${ }^{19}$ (fig. 2).

La hemodinámica cerebral tras la RCE se caracteriza por fallo en la reperfusión a nivel microcirculatorio, a pesar de una adecuada presión de perfusión cerebral (PPC), debido a la presencia de microtrombosis instauradas durante la parada cardíaca ${ }^{20}$. Sobre esto se añade reperfusión 


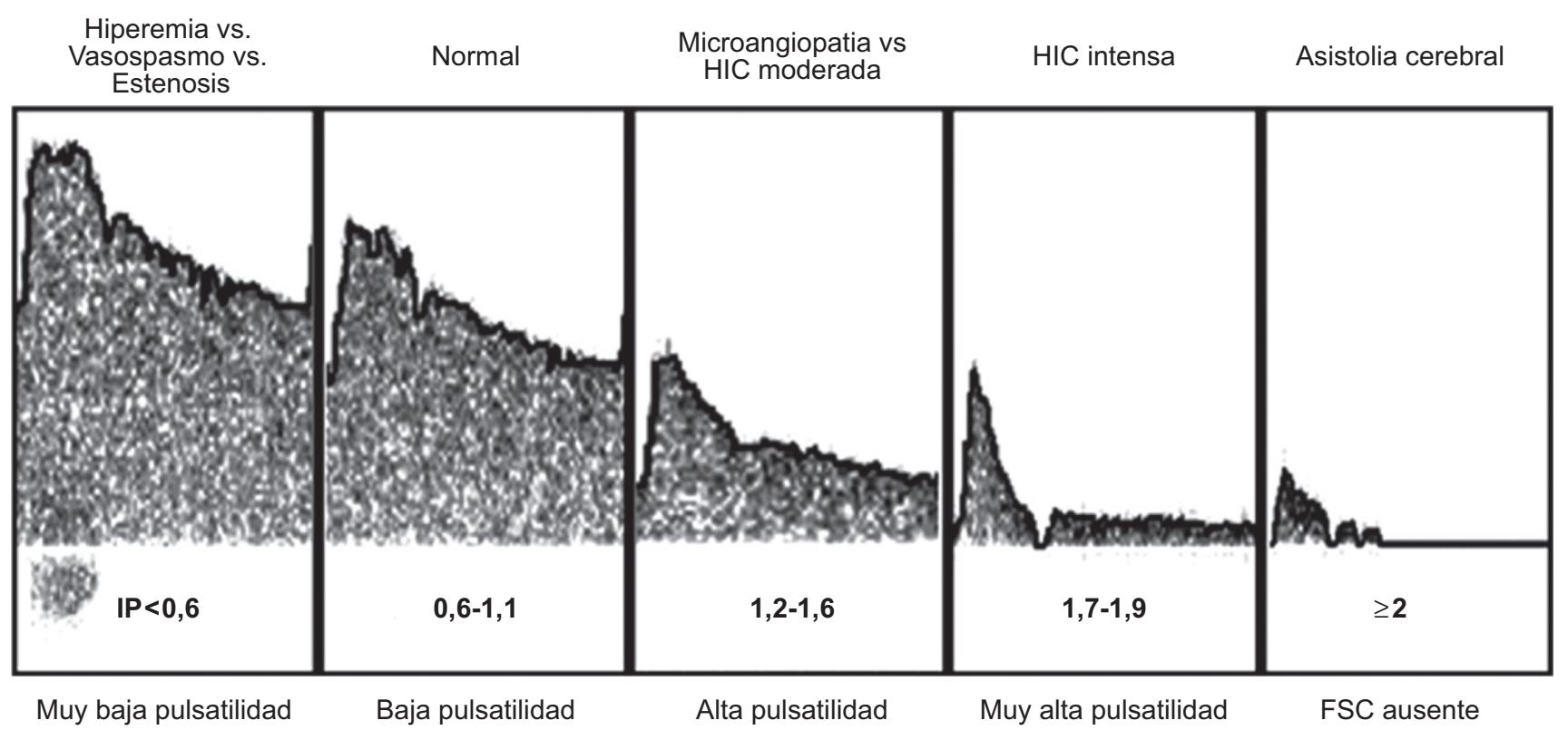

Figura 1 Posibilidades de espectros de velocidad del flujo sanguíneo cerebral con el Doppler transcraneal. HIC: hipertensión intracraneal; IP: índice de pulsatilidad.

hiperémica causada por aumento de la PPC y deterioro de la autorregulación cerebral, lo que producirá una mejoría inicial del $\mathrm{FSC}^{21}$, seguida a los 15 a $30 \mathrm{~min}$ de un estado de hipoperfusión cerebral que se añade al derivado de las microtrombosis ${ }^{22} \mathrm{y}$, posteriormente, a una exacerbación del edema y a daño por reperfusión ${ }^{23}$.

\section{Utilidad de la ultrasonografía Doppler transcraneal durante la resucitación}

Durante la RCP el gasto cardíaco cae por debajo del 25\% y el FSC cae a un 3-15\% en el caso de que las compresiones torácicas comiencen inmediatamente, y disminuye de forma progresiva durante los siguientes minutos ${ }^{24}$. El empleo de los ultrasonidos para la monitorización de los esfuerzos de resucitación durante la parada cardíaca es un concepto novedoso, sin duda merecedor de un comentario, pero todavía sin un peso de evidencia científica suficiente como para incluirse en las recomendaciones para su empleo sistemático.

El análisis de las alteraciones en la velocidad del flujo sanguíneo en las arterias del polígono de Willis, mediante la DTC, puede ser de gran utilidad al reflejar los cambios en la perfusión cerebral durante la parada cardíaca tratada con compresión torácica intermitente ${ }^{9,25}$. El verdadero desafío estriba en que la utilización de estas técnicas no suponga un excesivo aumento en la dificultad de realizar la RCP, por lo que se necesitaría adaptar los aparatos para un uso más específico dentro de la parada cardíaca (por ejemplo, con los cascos de monitorización bilateral multivaso disponibles en los modernos equipos de DTC) y un mayor número de estudios que pudieran confirmar de forma concluyente la validez de estos métodos de monitorización durante la RCP.

Durante la parada cardíaca las compresiones torácicas producen una apertura de las válvulas sigmoideas y un empuje del volumen sanguíneo que se halla en los

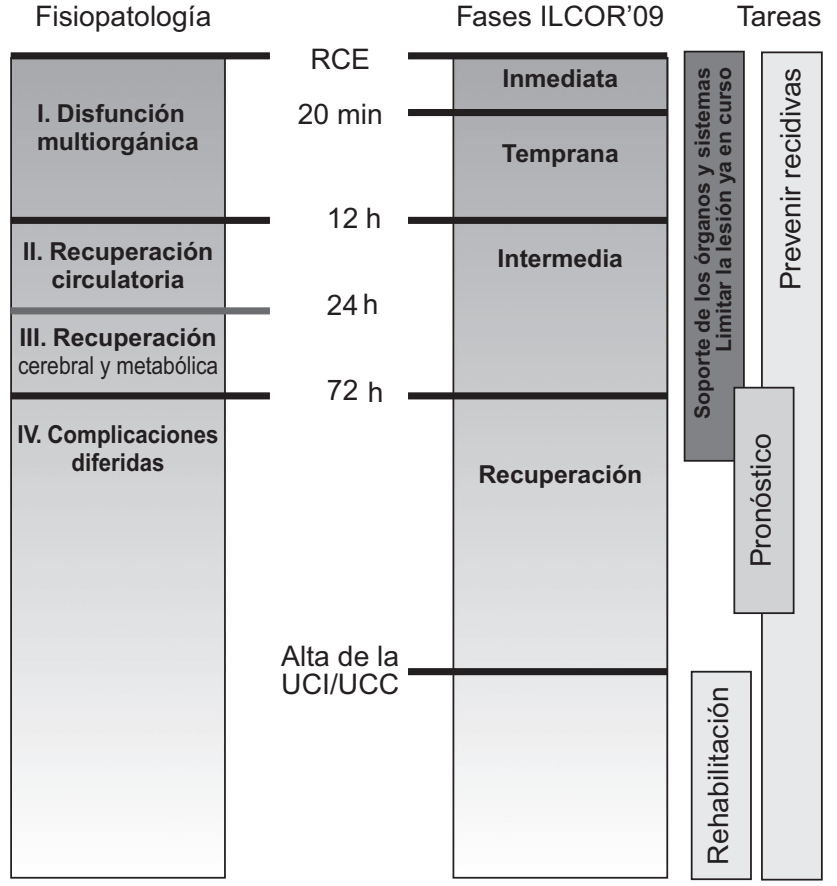

Figura 2 Correlación entre la fisiopatología y la evolución clínica.

ILCOR: International Liaison Committee on Resuscitation; RCE: recuperación de la circulación espontánea.

ventrículos, y se crea una sístole con su correspondiente volumen sistólico. Se producirá así un FSC característico que, según se ha podido constatar mediante la DTC, tan sólo existe durante la sístole y que refleja el ritmo y la intensidad de las compresiones torácicas, sin perfusión durante la diástole o esta es mínima y retrógrada (por debajo de la línea de base) (fig. 3). La Vm del FSC puede llegar inicialmente al $80 \%$ del valor normal, pero si el paciente 


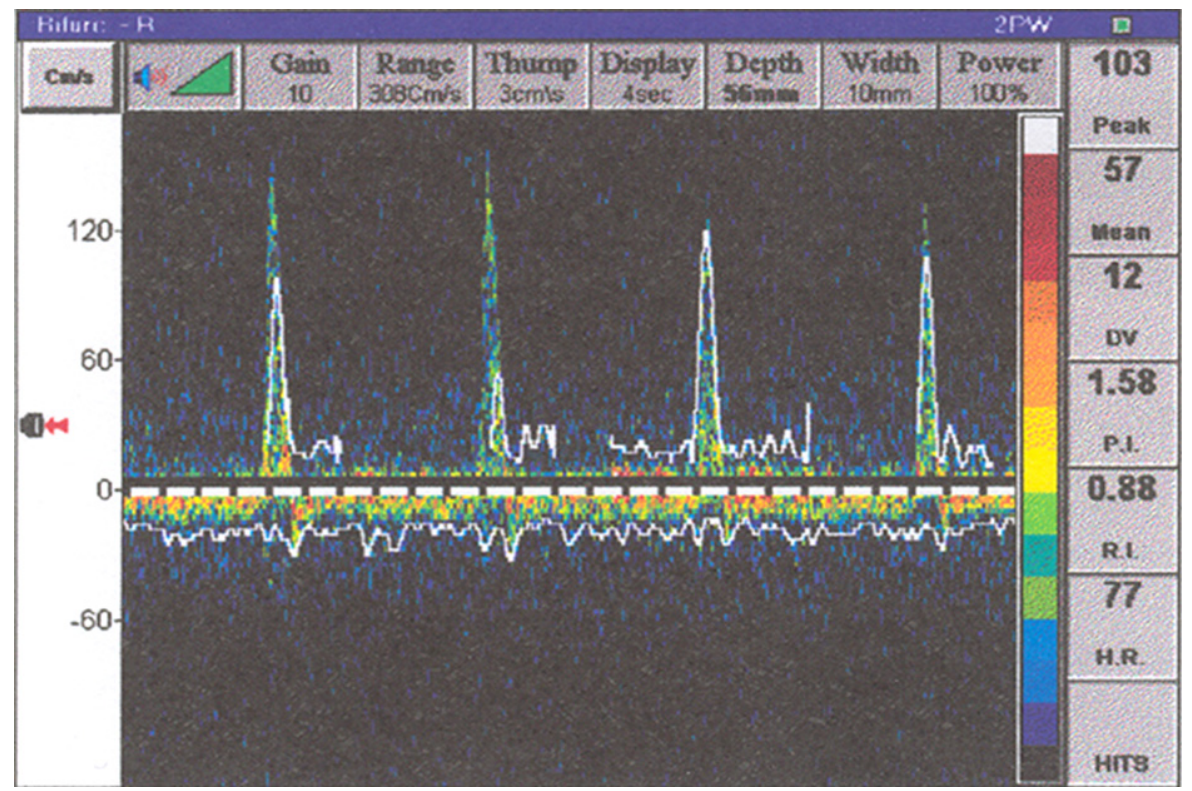

Figura 3 Espectro de la ultrasonografía Doppler transcraneal sin diástole o «espigado» durante las maniobras de resucitación cardiopulmonar.

no alcanza la RCE tendrá un deterioro progresivo durante la parada cardíaca y llegará con el tiempo a la ausencia total de flujo. Esto proporcionará una señal muy similar a la observada en la muerte encefálica ${ }^{26}$.

En estudios sobre animales durante la RCP se ha monitorizado simultáneamente la perfusión cerebral mediante 3 sistemas: un catéter bilateral a nivel de la arteria carótida interna para calcular la velocidad total del FSC, la DTC para registrar la VS y la Vm, y la cuantificación del FSC mediante microesferas radiactivas obtenidas post mórtem en el tejido cerebral tras haberse inyectado en la aurícula izquierda durante la RCP. Se pudo comprobar así que todos los valores descendían significativamente, se encontró una correlación significativa entre la Vm de la DTC y la Vm total en la carótida interna y, por otra parte, entre la VS de la DTC y el FSC cuantificado mediante microesferas ${ }^{9}$.

En cuanto al vaso ideal a insonar para la realización de la DTC durante la RCP parece que es el sifón de la arteria carótida interna a través de la ventana transorbitaria, por localizarse más consistentemente y proporcionar ondas con una morfología más óptima, ya que disminuye la incidencia de artefactos. Para obtener unos datos fiables se deben seguir una serie de premisas: a) usar una ganancia (ruido de fondo) lo suficientemente baja como para evitar la interferencia de artefactos, pero sin impedir el reconocimiento de la arteria; b) insonar ambas carótidas, ya que el flujo sanguíneo puede volverse heterogéneo, y se dan casos en los que una carótida ha doblado su flujo mientras la opuesta caía a la mitad, y c) usar un equipo de DTC que sea capaz de medir, en caso de que existiera, la onda diastólica retrógrada y usarla para calcular la $\mathrm{Vm}^{25}$.

La principal utilidad de la DTC durante la RCP es, por tanto, la monitorización del efecto de las compresiones torácicas sobre la perfusión cerebral para intentar optimizarla. Se ha evidenciado que las velocidades del FSC óptimas se mantienen tan solo durante $3 \mathrm{~min}$ a pesar de que el reanimador no refiera cansancio de manera indicativa y que la única maniobra que mejora de forma consistente las velocidades del FSC es el cambio de la persona que realiza las compresiones torácicas ${ }^{26}$. A pesar de esto, conforme se prolongue el tiempo de parada, la morfología de las ondas continuará empeorando sustancialmente. Lógicamente, la DTC podría facilitar también la evaluación de nuevas técnicas y procedimientos para mejorar la efectividad de las compresiones torácicas durante la RCP. La DTC puede aportar además información sobre el efecto de la administración de fármacos durante las maniobras de resucitación: tras la administración de bolos de suero fisiológico no se han observado cambios consistentes en la velocidad del FSC, tan sólo una mejoría de la señal Doppler durante 3 a $4 \mathrm{~s}$; sin embargo, la administración de adrenalina o bicarbonato puede aumentar la $\mathrm{Vm}$ a los $15 \mathrm{~s}$ en un $31-45 \%$ y durante los 30 s siguientes $^{26}$.

En cuanto a la resolución de la parada cardíaca, la RCE se registra inmediatamente mediante DTC, y se aprecia la recuperación de la perfusión cerebral espontánea al observarse un aumento de la velocidad del FSC, así como la aparición del componente diastólico del espectro, incluso antes de poder notarse la presencia de pulso arterial periférico. Como se ha comentado previamente, aquellos pacientes que no alcanzan la RCE en los siguientes minutos terminarán mostrando un patrón similar al de la muerte encefálica ${ }^{9,26}$.

\section{Valor de la ultrasonografía Doppler transcraneal tras la recuperación de la circulación espontánea}

Las manifestaciones clínicas del daño cerebral tras la RCE pueden incluir desde varios grados de disfunción cognitiva, convulsiones, mioclonías y coma, hasta la muerte encefálica. La menor vulnerabilidad o recuperación más precoz, tanto del diencéfalo como del tallo cerebral, serán los causantes de la gran tendencia de estos pacientes a quedar en estado vegetativo o en un estado de mínima consciencia. 
El pronóstico del coma hipóxico-isquémico tras la parada cardíaca es pobre, con una supervivencia del 10-25\% al final del primer año, de los que sólo un 3-10\% vuelven a su vida normal ${ }^{27}$.

La neuroprotección mediante hipotermia terapéutica a $32-34{ }^{\circ} \mathrm{C}$, iniciada precozmente y mantenida durante $24 \mathrm{~h}$, ha supuesto uno de los mayores avances en cuanto a la optimización de la recuperación neurológica tras la parada cardíaca y cambia radicalmente el pronóstico de pacientes que antes no habrían sobrevivido a este episodio o habrían quedado con graves secuelas ${ }^{28}$. Sin embargo, su aplicación correcta debe incluir una optimización hemodinámica que genera un incremento en el ya de por sí muy elevado consumo de recursos necesario para el tratamiento de estos pacientes en las UCI/UCC, y hace necesaria la disponibilidad de datos fiables para la monitorización y para el pronóstico de recuperación de estos pacientes ${ }^{29}$.

En el momento actual no se dispone de ninguna exploración neurológica tras la RCE que permita discernir qué pacientes van a tener una daño neurológico importante $\mathrm{e}^{30}$. Sólo el análisis de las circunstancias previas a la parada (no siempre conocidas en el momento de iniciar el tratamiento en una UCI/UCC) y los hallazgos de la exploración clínica neurológica transcurridas $72 \mathrm{~h}$ desde la RCE han mostrado tener algún valor pronóstico, pero tienen escasa utilidad clínica en el tratamiento individual de cada paciente, incluso al aplicar escalas de valoración multiparámetro ${ }^{31-33}$. Las técnicas de neuroimagen, pese a su valor predictivo en condiciones experimentales, han tenido hasta ahora muy escasa aplicabilidad en la práctica clínica ${ }^{18}$. Ciertos predictores oximétricos o bioquímicos de probada efectividad en condiciones de normotermia no se han validado hasta la fecha en hipotermia moderada ${ }^{34,35}$. Las pruebas neurofisiológicas sí parecen aportar datos sobre el pronóstico de recuperación, especialmente los potenciales evocados somatosensoriales que pueden hacerlo tras las primeras $24 \mathrm{~h}$, incluso con sedación e hipotermia ${ }^{36,37}$.

Aunque las recientes recomendaciones del International Liaison Committee on Resuscitation para el tratamiento del síndrome posparada cardíaca no hacen alusión alguna a la DTC $^{18}$, existe un interés creciente por la utilización de esta técnica de ultrasonidos como herramienta pronóstica y de monitorización tras la RCE ${ }^{13}$. Así, la DTC esta incluida entre las técnicas recomendadas en la plantilla Utstein para la recogida y la grabación de datos en el síndrome posparada cardíaca $^{38}$. La DTC permite identificar ciertos hallazgos iniciales y evolutivos que se han relacionado de forma concluyente con el pronóstico de estos pacientes y que tienen gran utilidad en su tratamiento asistencial, y es especialmente útil en situación de tratamiento con hipotermia, pues no ve interferidos sus registros por la modificación de la temperatura ${ }^{39}$.

\section{Hallazgos iniciales}

En los pacientes que se recuperan de una parada cardíaca durante al menos $20 \mathrm{~min}$, pero permanecen inconscientes, el patrón de la DTC inicial predominante incluye Vm bajas e IP altos («hipodinamia pulsátil»), y es reflejo de hipoperfusión secundaria a trombosis y vasoespasmo de la microcirculación cerebral, de hipoperfusión secundaria a la edematización celular y capilar o de ambas, y en ausencia de complicaciones, estos valores tenderán a normalizarse en las siguientes $72 \mathrm{~h}^{10}$. Este patrón se correlaciona con los hallazgos oximétricos y metabólicos registrados en humanos tras la recuperación de una parada cardíaca, con una baja extracción de oxígeno y una pobre actividad metabólica cerebral durante las primeras $2 \mathrm{~h}$ tras la $\mathrm{RCE}^{40}$. Sin embargo, debe tenerse en cuenta que la presencia de disfunción miocárdica intensa producirá por sí misma un descenso o, incluso, una práctica desaparición del componente diastólico del espectro Doppler sin que esto deba atribuirse a problemas en la microcirculación cerebral ${ }^{41}$.

La presencia inicial de un patrón hiperdinámico vendría definida por altas Vm en las arterias cerebrales medias y bajos IP, con cocientes $<3$ con las $\mathrm{Vm}$ registradas en el segmento distal submandibular de las arterias carótidas internas ipsilaterales. Este patrón podría registrarse «normalmente» hasta transcurridos 30 min desde la RCE y reflejar reperfusión macroscópica hiperémica causada por el aumento de la PPC y el deterioro de la autorregulación cerebral; sin embargo, por encima de 30 min su presencia se asocia de forma concluyente a mal pronóstico neurológico con evolución a hipertensión intracraneal ${ }^{42}$, facilitado entre otras razones por un descenso relevante en la presión crítica de cierre vascular ${ }^{43}$.

La presencia de un patrón de la DTC normal en esta primera valoración implica generalmente un buen pronósti$\mathrm{Co}^{42}$. Sin embargo, debe considerarse siempre en estos casos el estado de la autorregulación cerebral, medida habitualmente como reserva hemodinámica cerebral o reactividad vascular cerebral (RVC). Cuando la RVC es normal el pronóstico de recuperación neurológica tras la RCE suele ser muy favorable, pero si está disminuida, deberá tenerse un especial cuidado en el tratamiento hemodinámico y ventilatorio del paciente, ya que ante situaciones extremas de PPC se pueden producir fácilmente hiperemia (p. ej. por hipoventilación) o isquemia (p. ej. por hiperventilación) y ambas pueden complicar la evolución ${ }^{44}$. Para determinar esta RVC en el paciente resucitado de una parada cardíaca puede utilizarse el «test de respuesta hiperémica transitoria» o maniobra de Giller, que mide la autorregulación cerebral dinámica y analiza la modificación de la velocidad del FSC medida con DTC en las arterias cerebrales medias $(\mathrm{ACM})$ tras la realización durante $5 \mathrm{~s}$ de una compresión carotídea interna extracraneal ipsilateral; se considera normal cuando la relación entre la velocidad sistólica poscompresión y la basal (precompresión) es superior a $1,09^{45}$. En pacientes comatosos este test ha mostrado una correlación fiable con otros más complejos, como el de apnea-hiperventilación o el de inhalación de carbógeno ${ }^{46}$.

\section{Hallazgos evolutivos}

La DTC realizada a las 4,12 y $24 \mathrm{~h}$ tras la recuperación de una parada cardíaca permite detectar complicaciones e identificar a los pacientes con evolución a discapacidad muy intensa o fallecimiento ${ }^{10}$. La persistencia en estas determinaciones de hipodinamia (Vm bajas e IP elevados) indica una pérdida grave de la vasorregulación que se ha relacionado con una dependencia de la presión arterial sistólica para el flujo sanguíneo y con un incremento de la enolasa neuronal 

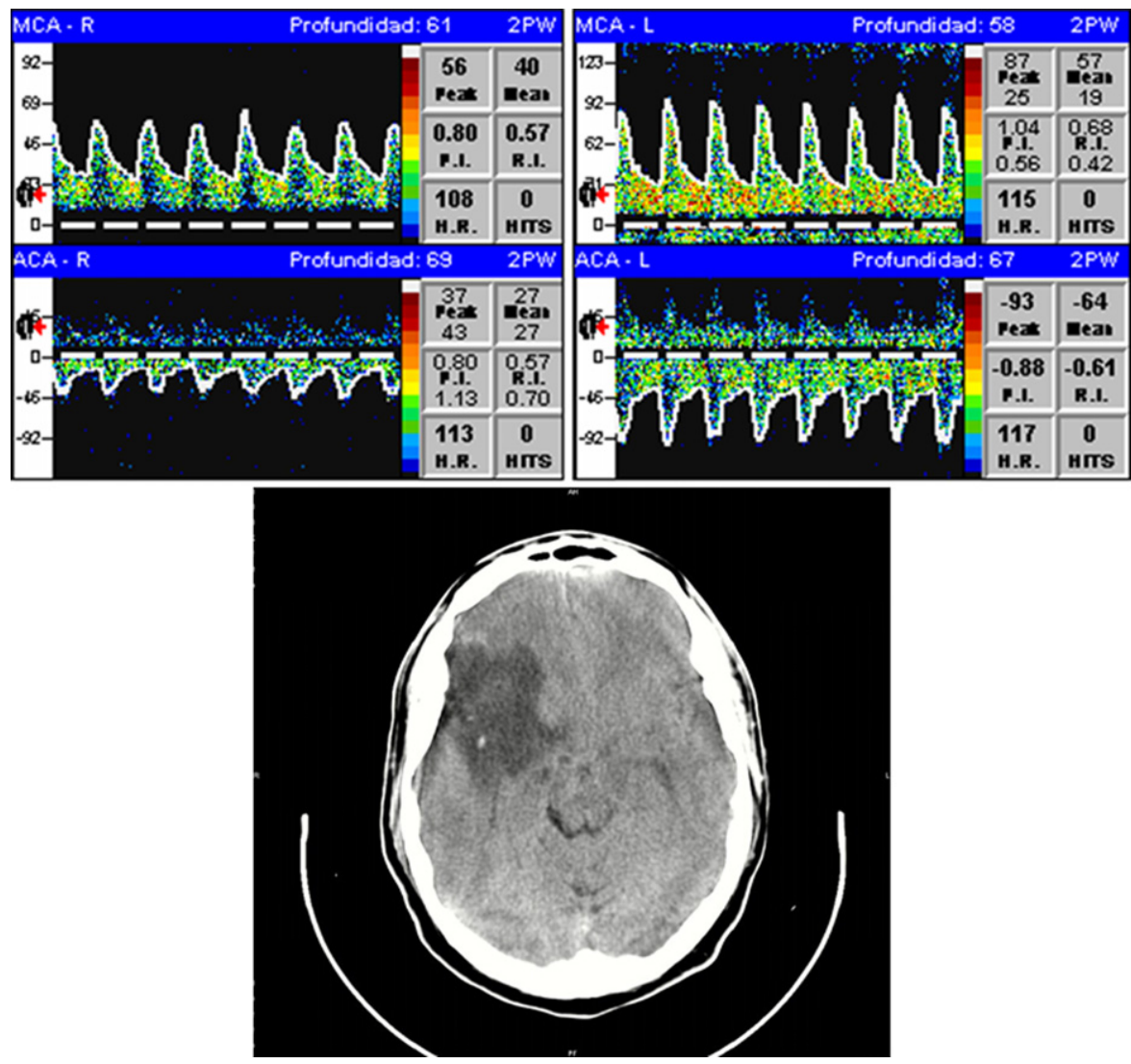

Figura 4 Hipodinamia relativa cerebral media y anterior derechas con normodinamia contralateral en la evolución de un paciente recuperado tras parada cardíaca. La TAC confirmó la presencia de un infarto isquémico frontoparietal derecho.

específica, todo esto indicador de muy mal pronóstico ${ }^{47}$. Un patrón de la DTC hipodinámico puede ser también consecuencia de una hiperemia previa con evolución desfavorable hacia hipertensión intracraneal. Como ya se mencionó anteriormente, en ambos casos deberá descartarse la presencia de disfunción miocárdica intensa productora de un descenso en el componente diastólico del espectro de la DTC $^{41}$.

Transcurridas $12 \mathrm{~h}$ desde la RCE se puede detectar también con la DTC la aparición en las arterias cerebrales de un patrón de hiperemia tardía caracterizado por una Vm alta con IP bajo y un cociente $<3$ entre Vm en ACM y Vm en el segmento distal extracraneal de la arteria carótida interna (ACl-DIST), de no tratarse, esta hiperemia puede llevar a hipertensión intracraneal, con transformación progresiva del patrón de la DTC en hipodinamia de alta pulsatilidad y frecuente evolución a asistolia cerebral ${ }^{48}$. Al hacer un paralelismo con los pacientes tratados con hipotermia terapéutica moderada por traumatismo craneoencefálico, esta hiperemia sería también un precursor de edema cerebral y su aparición en la fase de recalentamiento debería llevar a la inmediata suspensión y a la reinstauración de la hipotermia moderada ${ }^{49}$.

Durante la evolución del paciente son aplicables también los comentarios anteriores sobre el cálculo de la RVC mediante el test de respuesta hiperémica transitoria en situaciones de patrón DTC normodinámico, pues la alteración de la autorregulación puede aparecer en la evolución del paciente y no estar presente desde la $\mathrm{RCE}^{46}$. Por último, el hallazgo evolutivo de la presencia de arterias cerebrales hipodinámicas, alternadas con otras con patrón DTC normal o hiperémico, puede indicar la presencia de hipoperfusión focal y se ha indicado como predictor de ictus tras la parada cardíaca recuperada $^{50}$ (fig. 4).

\section{Detección y tratamiento de complicaciones}

Transcurridas $24 \mathrm{~h}$ desde la RCE se entra en el período de riesgo de aparición de complicaciones diferidas, por lo que el paciente resucitado deberá considerarse a todos los efectos como un paciente neurocrítico. La utilidad de la DTC en estos pacientes esta hoy fuera de toda duda. En un estudio realizado en España se comprobó que la realización diaria de determinaciones con DTC influyó en el tratamiento del 36\% de los pacientes ${ }^{51}$, y es muy probable que esta utilidad se vea incrementada al aumentar la frecuencia de las determinaciones y al extender sus indicaciones en línea con las nuevas tendencias de neuromonitorización en pacientes críticos ${ }^{52-54}$.

Puede tener especial interés en estos pacientes la aplicación de la DTC para el diagnóstico de la hipertensión intracraneal. La evolución de un patrón de la DTC hiperdinámico inicial hacia una morfología hipodinámica con disminución de la Vm y aumento de la pulsatilidad es un indicador no invasivo de hipertensión intracraneal y su monitorización en la práctica clínica es equivalente a la obtenida con los métodos invasivos ${ }^{55,56}$. El efecto del tratamiento de estos pacientes también puede monitorizarse 
y existe una excelente correlación entre los cambios en la velocidad del FSC medidos por DTC en las arterias cerebrales medias y los descensos del FSC comprobados en pruebas más complejas, como la resonancia magnética funcional ${ }^{14}$.

El mayor intervencionismo diagnóstico y terapéutico que caracteriza el tratamiento actual de los pacientes tras la RCE puede llevar implícita una mayor presencia de complicaciones no observadas anteriormente. Merecen especialmente destacarse las relacionadas con la aplicación de tratamientos fibrinolíticos, antiagregantes y anticoagulantes, que pueden llevar a la aparición de hemorragias intracraneales cuyos efectos sobre la presión intracraneal son fácilmente detectables mediante DTC (patrón de hipodinamia de alta pulsatilidad) como también lo es la aparición de vasoespasmo arterial (patrón de hiperdinamia de baja pulsatilidad y cociente $>3$ entre Vm en ACM y Vm en $\mathrm{ACl}$-DIST) que puede producir isquemia focal e ictus, y es también de interés la aplicación de la DTC en la monitorización de su tratamiento ${ }^{57}$.

Además de la ya comentada utilidad de la DTC para pronosticar ictus en pacientes con distribución heterogénea del FSC tras la RCE ${ }^{50}$, los pacientes pueden tener episodios isquémicos cerebrales agudos de cualquier otra causa, especialmente por la coexistencia de otros factores de riesgo vascular además de los que pudieron provocar la parada cardíaca (enfermedad carotídea, fibrilación auricular, foramen oval permeable, etc. $)^{58}$. La DTC ha mostrado también utilidad para el diagnóstico temprano de estos episodios $y$ ha llegado a convertirse incluso en una herramienta esencial para su tratamiento ${ }^{59}$.

La DTC es sin duda una herramienta para conseguir «vida encefálica» ${ }^{60}$. Sin embargo, la evolución de una hiperemia que complique el síndrome posparada cardíaca puede llevar a hipertensión intracraneal refractaria y asistolia cerebral. En estas circunstancias, la DTC es hoy día el único método no invasivo realizado a la cabecera del paciente que es fiable en presencia de sedantes e hipotermia para realizar precozmente el diagnóstico de muerte encefálica. Es necesario, no obstante, recordar que deben cumplirse unos requerimientos de entrenamiento y equipamiento en el equipo asistencial y que, en ausencia de lesión estructural evidente, pueden observarse patrones de flujo sin función o flujos de transición que desaparecerán si se prolonga el período de observación ${ }^{61}$.

Para la utilización de la DTC como apoyo diagnóstico de muerte encefálica es necesario documentar la existencia de flujo reverberante, espigas sistólicas o ausencia de señal en ambas ACM y en la arteria basilar, aunque se recomienda también evaluar el flujo en el resto de las arterias del polígono de Willis; el registro debe mantenerse al menos durante $30 \mathrm{~s}$ por arteria insonada, registrarse en papel o soporte recuperable y confirmar los datos mediante un segundo estudio realizado al cabo de al menos $30 \mathrm{~min}$. El uso en estos casos de sustancias ecopotenciadoras de la señal de la DTC puede salvar la ausencia de ventana acústica y posibilitar la localización de flujos de mínima intensidad ${ }^{62}$.

\section{Conclusión}

El actual enfoque de la resucitación «orientado hacia el flujo sanguíneo» requiere de herramientas fiables de monitorización.
La DTC tiene gran utilidad en resucitación y permite primero guiar los esfuerzos de los reanimadores para optimizar el tratamiento de la parada cardíaca y después los esfuerzos de los equipos asistenciales para el tratamiento del síndrome posparada cardíaca. La calidad de la RCP puede ser medida «latido a latido» en términos de velocidad sistólica máxima del FSC alcanzada, tanto con las compresiones torácicas manuales como con las realizadas por dispositivos mecánicos, y también puede medirse de esta forma el impacto de las medias farmacológicas de soporte vital avanzado. La utilización de exámenes de la DTC seriados tras la RCE permite detectar y tratar precozmente las alteraciones en la hemodinámica cerebral, y disminuye la probabilidad de daño neurológico secundario. La DTC identifica en las primeras $24 \mathrm{~h}$ a los pacientes que han evolucionado hacia un daño neurológico irreversible, evita así la futilidad terapéutica, y tiene el valor añadido de no verse interferido en sus registros por la utilización de sedantes o por el tratamiento mediante hipotermia moderada.

\section{Conflicto de intereses}

Los autores declaran no tener ningún conflicto de intereses.

\section{Bibliografía}

1. Álvarez-Fernández JA, Gazmuri RJ. Mortalidad evitable por parada cardíaca extrahospitalaria. Med Clin (Barc). 2008;130: $710-4$.

2. Nadkarni V, Larkin G, Peberdy M, Carey SM, Kaye W, Mancini ME, et al. First documented rhythm and clinical outcome from inhospital cardiac arrest among children and adults. JAMA. 2006;295:50-7.

3. Gazmuri RJ, Álvarez-Fernández JA. Tendencias en resucitación cardiopulmonar. Med Intensiva. 2009;33:31-9.

4. Nolan JP, Morley PT, Hoek TL, Hickey RW. Advancement life support task force of the International Liaison committee on Resuscitation. Therapeutic hypothermia after cardiac arrest. An advisory statement by the Advancement Life support Task Force of the International Liaison committee on Resuscitation. Resuscitation. 2003;57:231-5.

5. Gaieski DF, Band RA, Abella BS, Neumar RW, Fuchs BD, Kolansky DM, et al. Early goal-directed hemodynamic optimization combined with therapeutic hypothermia in comatose survivors of out-ofhospital cardiac arrest. Resuscitation. 2009;80:418-24.

6. Gazmuri RJ, Kube E. Capnography during cardiac resuscitation: A clue on mechanisms and a guide to interventions. Crit Care. 2003;7:R139-44.

7. Hayes MM, Berg RA, Otto CW. Monitoring during cardiac arrest: Are we there yet? Curr Opin Crit Care. 2003;9:211-7.

8. Mörtberg E, Cumming P, Wiklund L, Wall A, Rubertsson S. A PET study of regional cerebral blood flow after experimental cardiopulmonary resuscitation. Resuscitation. 2007;75:98-104.

9. Lewis LM, Stothert Jr JC, Gómez CR, Ruoff BE, Hall IS, Chandel B, et al. A noninvasive method for monitoring cerebral perfusion during cardiopulmonary resuscitation. J Crit Care. 1994;9:169-74.

10. Lemiale $V$, Huet $O$, Vigué $B$, Mathonnet A, Spaulding C, Mira JP, et al. Changes in cerebral blood flow and oxygen extraction during post-resuscitation syndrome. Resuscitation. 2008;76: $17-24$.

11. Aaslid R, Markwalder TM, Nornes H. Noninvasive transcranial Doppler ultrasound recording of flow velocity in basal cerebral arteries. J Neurosurg. 1982;57:769-74.

12. Baumgartner RW. Transcranial color-coded duplex sonography. J Neurol. 1999;246:637-47. 
13. Álvarez-Fernández JA, Pérez-Quintero R. Use of transcranial Doppler ultrasound in the management of post-cardiac arrest syndrome. Resuscitation. 2009;80:1321-2.

14. Valdueza JM, Balzer JO, Villringer A, Vogl TJ, Kutter R, Einhaüpl $\mathrm{KM}$. Changes in blood flow velocity and diameter of the middle cerebral artery during hyperventilation: Assessment with MR and transcranial Doppler sonography. AJNR. 1997;18:1929-34.

15. Fukushima U, Sasaki S, Okano S, Takase K, Hagio M. The comparison between the cerebral blood flow directly measures and cerebral blood flow velocity in the middle and basilar cerebral arteries measured by transcranial Doppler ultrasonography. J Vet Med Sci. 1999;61:1293-7.

16. Laver S, Farrow C, Turner D, Nolan J. Mode of death after admission to an intensive care unit following cardiac arrest. Intensive Care Med. 2004;30:2126-8.

17. Gopalan KT, Lee J, Ikeda S, Burch CM. Cerebral blood flow velocity during repeatedly induced ventricular fibrillation. J Clin Anesth. 1999;11:290-5.

18. Nolan JP, Neumar RW, Adrie C, Aibiki M, Berg RA, Böttiger BW, et al. Post-cardiac arrest syndrome: Epidemiology, pathophysiology, treatment, and prognostication. A Scientific Statement from the International Liaison Committee on Resuscitation; the American Heart Association Emergency Cardiovascular Care Committee; the Council on Cardiovascular Surgery and Anesthesia; the Council on Cardiopulmonary, Perioperative, and Critical Care; the Council on Clinical Cardiology; the Council on Stroke. Resuscitation. 2008;79:350-79.

19. Negovsky VA, Gurvitch AM. Post-resuscitation disease-a new nosological entity. Its reality and significance. Resuscitation. 1995;30:23-7.

20. Fischer M, Böttiger BW, Popov-Cenic S, Hossmann KA. Thrombolysis using plasminogen activator and heparin reduces cerebral no-reflow after resuscitation from cardiac arrest: An experimental study in the cat. Intensive Care Med. 1996;22:1214-23.

21. Sundgreen C, Larsen FS, Herzog TM, Knudsen GM, Boesgaard S, Aldershvile J. Autoregulation of cerebral blood flow in patients resuscitated from cardiac arrest. Stroke. 2001;32:128-32.

22. Wolfson Jr. SK, Safar P, Reich H, Clark JM, Gur D, Stezoski W, et al. Dynamic heterogeneity of cerebral hypoperfusion after prolonged cardiac arrest in dogs measured by the stable xenon/ CT technique: A preliminary study. Resuscitation. 1992;23:1-20.

23. Müllner M, Sterz F, Binder M, Hellwagner K, Meron G, Herkner H, et al. Arterial blood pressure after human cardiac arrest and neurological recovery. Stroke. 1996;27:59-62.

24. Paraskos JA, Orquiola AA. Cardiopulmonary resuscitation. En: Irwin RS, Rippe JM, editores. Irwin and Rippe's Intensive Care Medicine, 6 ed. Philadelphia: Lippincott Williams \& Wilkins; 2008. p. 182-211.

25. Lewis LM, Stothert Jr JC, Kraus GE, Gómez CR, Goodgold H, Keltner Jr RM, et al. A comparison of transcranial Doppler ultrasound (TCD) and radioactive microspheres in determining cerebral perfusion in normal and low flow states. Resuscitation. 1990;20:213-20.

26. Lewis LM, Gómez CR, Ruoff BE, Gómez SM, Hall IS, Gasirowski B. Transcranial Doppler determination of cerebral perfusion inpatients undergoing CPR: Methodology and preliminary findings. Ann Emerg Med. 1990;19:1148-51.

27. Ruscalleda J, Sanromán L, De Juan M, Guardia E. Paciente neurocrítico y paro cardiorrespiratorio. Valor diagnóstico y pronóstico de la tomografía computerizada y la resonancia magnética. En: Net A, Marruecos-Sant L, editores. El paciente neurocrítico. Barcelona: Ars Médica; 2006. p. 1-15.

28. Hypothermia after Cardiac Arrest Study Group. Mild therapeutic hypothermia to improve the neurologic outcome after cardiac arrest. N Engl J Med. 2002;346:549-56.

29. Álvarez-Fernández JA, Pérez-Quintero R. Transcranial Doppler ultrasound in therapeutic hypothermia for cardiac arrest survivors. Crit Care Med 2010 (En prensa).
30. Booth CM, Boone RH, Tomlinson G, Detsky AS. Is this patient dead, vegetative, or severely neurologically impaired? JAMA. 2004;291:870-9.

31. Wijdicks E, Hijdra A, Young G, Bassetti CL, Wiebe S. Practice parameter: Prediction of outcome in comatose survivors after cardiopulmonary resuscitation (an evidence-based review). Neurology. 2006;67:203-10.

32. Geocadin RG, Eleff SM. Cardiac arrest resuscitation: Neurologic prognostication and brain death. Curr Opin Crit Care. 2008;14: 261-8.

33. Wijdicks EF, Bamlet WR, Maramattom BV, Manno EM, McClelland RL. Validation of a new coma scale: The FOUR score. Ann Neurol. 2006;60:744-5.

34. Zarzuelo R, Castañeda J. Differences in oxygen content between mixed venous blood and cerebral venous blood for outcome prediction after cardiac arrest. Intensive Care Med. 1995;21:71-5.

35. Takasu A, Yagi K, Ishihara S, Okada Y. Combined continuous monitoring of systemic and cerebral oxygen metabolism after cardiac arrest. Resuscitation. 1995;29:189-94.

36. Fischer C, Luauté J, Némoz C, Morlet D, Kirkorian G, Mauguière F. Improved prediction of awakening or nonawakening from severe anoxic coma using tree-based classification analysis. Crit Care Med. 2006;34:1520-4.

37. Zandbergen EGJ, Koelman HTM, Haan RJ, Hijdra A, for the PROPAC-Study Group. SEEPs and prognosis in postanoxic coma: Only short or also long latency responses? Neurology. 2006;67: 583-6.

38. Langhelle A, Nolan J, Herlitz J, Castren M, Wenzel V, Soreide E, et al. On behalf of the participants at the 2003 Utstein Consensus Symposium. Recommended guidelines for reviewing, reporting, and conducting research on postresuscitation care: The Utstein style. Resuscitation. 2005;66: 271-83.

39. Wennervirta JE, Ermes MJ, Tiainen SM, Salmi TK, Hynninen MS, Särkelä MO, et al. Hypothermia-treated cardiac arrest patients with good neurological outcome differ early in quantitative variables of EEG suppression and epileptiform activity. Crit Care Med. 2009;37:2427-35.

40. Gómez CR, McLaughlin JR, Njemanze PC, Nashed A. Effect of cardiac dysfunction upon diastolic cerebral blood flow. Angiology. 1992;43:625-30.

41. Beckstead JE, Tweed WA, Lee J, MacKeen WL. Cerebral blood flow and metabolism in man following cardiac arrest. Stroke. 1978;9:569-73.

42. Nebra-Puertas AC, Virgós-Señor V, Suárez-Pinilla MA, MunárrizHinojosa J, Ridruejo-Sáez R, Sánchez-Miret JI, et al. Modificaciones en la velocidad de flujo cerebral medidas mediante Doppler transcraneal, tras maniobras de soporte vital avanzado. Med Intensiva. 2003;27:219-23.

43. Aaslid R, Lash SR, Bardy GH, Gild WH, Newell DW. Dynamic pressure-flow velocity relationships in the human cerebral circulation. Stroke. 2003;34:1645-9.

44. Ng SC, Poon WS, Chan MT, Lam JM, Lam W, Metreweli C. Transcranial Doppler ultrasonography (TCD) in ventilated head injured patients: Correlation with stable xenon-enhanced CT. Acta Neurochir Suppl. 2000;76:479-82.

45. Giller CA. A bedside test for cerebral autoregulation using transcranial Doppler ultrasound. Acta Neurochir (Wien). 1991;108:7-14.

46. Buunk G, van der Hoeven JG, Meinders AE. Cerebrovascular reactivity in comatose patients resuscitated from a cardiac arrest. Stroke. 1997;28:1569-73.

47. Wessels T, Harrer JU, Jacke C, Janssens U, Klötzsch C. The prognostic value of early transcranial Doppler ultrasound following cardiopulmonary resuscitation. Ultrasound Med Biol. 2006;32:1845-51. 
48. Iida K, Satoh H, Arita K, Nakahara T, Kurisu K, Ohtani M. Delayed hyperemia causing intracranial hypertension after cardiopulmonary resuscitation. Crit Care Med. 1997;25:971-6.

49. Iida K, Kurisu K, Arita K, Ohtani M. Hyperemia prior to acute brain swelling during rewarming of patients who have been treated with moderate hypothermia for severe head injuries. J Neurosurg. 2003;98:793-9.

50. Carbutti G, Romand JA, Carballo JS, Bendjelid S, Suter PM, Bendjelid K. Transcranial Doppler: An early predictor of ischemic stroke after cardiac arrest? Anesth Analg. 2003;97:1262-5.

51. Grupo de Trabajo de Neurointensivismo y Trauma de la Sociedad Española de Medicina Intensiva. Crítica y Unidades Coronarias (SEMICYUC) y Grupo de Trabajo de Neurología Crítica de la Societat Catalana de Medicina Intensiva i Crítics (SOCMIC). Utilidad clínica de un registro diario de Doppler transcraneal en el paciente neurológico agudo. Estudio multicéntrico. Med Clin (Barc). 2003;120:241-5.

52. White H, Venkatesh B. Applications of transcranial Doppler in the ICU: A review. Intensive Care Med. 2006;32:981-94.

53. Álvarez-Fernández JA, Pérez Quintero R. Some more applications of transcranial Doppler in the ICU. Intensive Care Méd. 2007;33:1667-8.

54. Rasulo FA, De Peri E, Lavinio A. Transcranial Doppler ultrasonography in intensive care. Eur J Anaesthesiol Suppl. 2008;42: 167-73.
55. Schmidt B, Czosnyka M, Klingelhöfer J. Clinical applications of a non-invasive ICP monitoring method. Eur J Ultrasound. 2002;16: $37-45$.

56. Bellner J, Romner B, Reinstrup P, Kristiansson KA, Ryding E, Brandt L. Transcranial Doppler sonography pulsatility index (PI) reflects intracranial pressure (ICP). Surg Neurol. 2004;62:45-51.

57. Schatlo B, Pluta RM. Clinical applications of transcranial Doppler sonography. Rev Recent Clin Trials. 2007;2:49-57.

58. Garami Z, Alexandrov AV. Neurosonology. Neurol Clin. 2008;27: 89-108.

59. Alexandrov AV, Molina CA, Grotta JC, Garami Z, Ford SR, Álvarez-Sabin J, et al. CLOTBUST Investigators. Ultrasoundenhanced systemic thrombolysis for acute ischemic stroke. $\mathrm{N}$ Engl J Med. 2004;351:2170-8.

60. Álvarez Fernández JA, Pérez-Quintero R. Ultrasonidos y vida encefálica. Med Intensiva. 2006;30:113-5.

61. Calleja S, Tembl JI, Segura T. Por la Sociedad Española de Neurología. Recomendaciones sobre el uso del Doppler transcraneal para determinar la existencia de paro circulatorio cerebral como apoyo diagnóstico de la muerte encefálica. Neurología. 2007;22:441-7.

62. Abadal JM, Llompart-Pou JA, Homar J, Pérez-Bárcena J, Ibáñez J. Aplicaciones del dúplex transcraneal codificado en color en la monitorización del enfermo neurocrítico. Med Intensiva. 2007;31:510-7. 